\title{
Editorial
}

\section{Combined technique for cardiac anaesthesia}

With the recent renewed interest in early extubation after cardiac surgery, it now behooves the anaesthetist to modify the more traditional cardiac anaesthetic technique to fit this aim. However early extubation depends on an anaesthetic technique that combines both good intraoperative anaesthesia, producing haemodynamic stability, with profound postoperative analgesia. The latter is particularly important as pain is the primary cause of postoperative pulmonary complications. ${ }^{1}$

The idea of early extubation after cardiac surgery was first suggested in the mid 1970's when an inhalational anaesthetic technique was popular. ${ }^{2}$ Prolonged postoperative support was then, (and still is) routine after cardiac surgery in many centres to reduce hypoxia and pulmonary complications. ${ }^{3}$ With popularization of the high dose opioid technique in the late 1970 's, ${ }^{4}$ especially with the introduction of fentanyl, ${ }^{5}$ postoperative respiratory support became mandatory. Recently improvements in anaesthetic, surgical, monitoring and extracorporeal circulatory techniques have considerably reduced the incidence of postoperative respiratory complications. ${ }^{6}$ This has lead to a reevaluation of the need for delayed extubation.

The possibility of safe and early extubation depends on the anaesthetic methods used for intraoperative anaesthesia and postoperative analgesia. Recently, much interest has been focused on a technique developed by Cheng $e t$ al. in Toronto ${ }^{7}$ which uses short acting agents during the intraoperative course and conventional on demand analgesia postoperatively. In this issue of the Journal Kowalenski et al. ${ }^{8}$ describe a technique of general anaesthesia supplemented with subarachnoid bupivacaine and morphine in 18 patients undergoing coronary artery bypass surgery. The aims of this innovative technique were to provide a stable intraoperative haemodynamic course, to decrease requirements for long-acting opioids and, potentially, to obtain the beneficial effects of cardiac sympathectomy. After general anaesthesia with alfentanil $97 \pm 22 \mu \mathrm{g} \cdot \mathrm{kg}^{-1}$ and midazolam $0.04 \pm 0.02 \mathrm{mg} \cdot \mathrm{kg}^{-1}$, subarachnoid anaesthesia (SA) with hyperbaric bupivacaine $23-30 \mathrm{mg}$ ( 15 patients), lidocaine $150 \mathrm{mg}$ (1 patient)

From the Department of Anaesthesia, Royal Victoria Hospital, Montreal, Quebec.
Fiona E. Ralley $\mathrm{MBChB}$ FRCA or a combination ( 2 patients), and unpreserved morphine $0.5-1.0 \mathrm{mg}$ was administered using either a 25 ga or 22 ga needle. Although 17 patients received phenylephrine at some time during their intraoperative course it was not specifically related to the administration of SA. (7 patients after induction, 8 patients after SA, 13 patients during CPB and 7 patients after CPB). Time to awakening was $1.6 \pm 1.1 \mathrm{hr}$ after arrival in the ICU. In nine patients $(50 \%)$ the trachea was extubated on the day of surgery (mean extubation time $6.3 \pm 1.2 \mathrm{hr}$ ). This really represents $75 \%$ of the possible patients as $6 / 18$ were ventilated electively overnight due to their ICU practice of overnight ventilation for all afternoon cases. Of significance five patients received no additional analgesia before extubation. Three patients received opioid infusions for overnight ventilation and the other ten patients received total doses of morphine 2-14 $\mathrm{mg} \dot{i v}$ and/or fentanyl $100-500 \mu \mathrm{g} i v$.

Did the authors achieve their goals? As stated by the authors, the retrospective nature of their report is an obvious weakness. Its major deficiency being their inability to quantitate precisely haemodynamic changes, therefore making it difficult to demonstrate whether a stable intraoperative haemodynamic course was achieved. Nonetheless, we are assured by them that their seemingly high use of phenylephrine was common in their institution. Without the luxury of a control group with which to draw comparison it is difficult to make any judgement. Their statement that induction of SA produced little additional haemodynamic change needs to be better substantiated than by the data they present in this issue. Eight of 18 patients requiring vasoactive drugs after SA. Certainly the dose of alfentanil used intraoperatively was much less than that reported by others. ${ }^{9}$ Their incidence of $16 \%$ patients responding to sternotomy compares favourably with the $20 \%$ reported by Robbins et al. ${ }^{9}$ where ten times the dose of alfentanil was administered. This, associated with the low concentration of isoflurane $(<0.5$ MAC), suggests that the spinal anaesthetic contributed considerably to the total anaesthetic technique. Whether cardiac sympathectomy was achieved is unknown. Some degree of sympathetic blockade was produced, but as no anaesthetic level was recorded we are left to speculate 
its extent. Despite these negative comments the data indicates that this technique is worth further investigation. It is rather the retrospective method of reporting that is to be criticised more than the data. A properly designed prospective study is to be encouraged.

Previous studies using neuraxial analgesia in patients undergoing cardiac surgery have generally used the epidural route. ${ }^{10,11}$ Controversy still exists about the use of thoracic epidural anaesthesia (TEA) in patients for surgery requiring full heparinization. The major concern being the risk of epidural haemorrhage and haematoma with serious neurological sequelae. As yet, there is no absolute answer to the question of the safety of performing epidural or spinal anaesthesia immediately before full anticoagulation. However, as more and more studies are published showing that the incidence of neurological sequelae associated with these techniques is extremely low, they will become more popular. In a study by Liem $e t$ $a l .{ }^{11}$ the use of high TEA during CABG surgery for postoperative pain management was compared with intermittent intravenous opioid administration. In an attempt to prevent neurological complications, the authors required normal coagulation parameters. Therefore in all their patients, aspirin therapy was stopped ten days before surgery. Furthermore, the epidural catheter was inserted at least $20 \mathrm{hr}$ preoperatively. Patients in the TEA group were awake sooner, resumed spontaneous respiration earlier and their tracheas were extubated earlier. In addition their visual analog pain scores and sedation scores were significantly better.

Despite these obvious advantages, two major disadvantages to the technique exist. First, with the increasing trend to reduce the preoperative time patients are in hospital prior to surgery, to the point of same day admission even for cardiac surgery patients, the need to insert the catheter nearly $24 \mathrm{hr}$ preoperatively may not be feasible in many institutions. Second, many cardiologists would not be happy at the request to stop aspirin so long before surgery, and this would also preclude the use of this technique in many patients who require urgent or emergency surgery. Therefore such a strictly cautious protocol obviously limits the use of epidural anaesthesia for cardiac surgery. Inherently one would suppose that the use of the smaller 27 ga spinal needle compared with the 16 ga epidural needle would reduce the risk of haematoma formation postoperatively. Unfortunately this has never been studied. Although recent studies report the apparent safety of neuraxial analgesia in patients receiving anticoagulants, ${ }^{12}$ one should continue to maintain a high degree of awareness of the possibility of epidural haematoma and the importance of periodical neurological examination.

With the ever continuing search for the perfect cardiac anaesthetic producing optimal haemodynamic stability during the prebypass period, perhaps a new era may be evolving: a combined regional with general technique. The traditional high dose cardiac anaesthetic is rapidly becoming a thing of the past as outcome studies show little difference among different anaesthetic techniques: rather the importance in maintaining haemodynamic stability throughout the surgery. It is hoped that the report in this issue ${ }^{8}$ will stimulate further investigation into both the safety and efficacy of this novel approach to anaesthesia for patients for cardiac surgery.

\section{Les techniques combinées en anesthésie cardiaque}

L'intérêt renouvelé qu'il accorde à l'extubation précoce après la chirurgie cardiaque oblige l'anesthésiste à revoir ses méthodes traditionelles. L'extubation précoce nécessite une technique qui associe anesthésie efficace et hémodynamie stable pendant l'intervention avec analgésie profonde après l'intervention. Cette dernière condition est particulièrement importante parce que la douleur constitue la cause principale des complications qui surviennent après l'intervention. '

Le concept de l'extubation précoce après la chirurgie cardiaque a d'abord été mis de l'avant au milieu des années 1970 alors que les techniques inhalatoires étaient populaires. ${ }^{2}$ Une assistance respiratoire prolongée était (et est toujours) la règle après la chirurgie cardiaque dans bien des centres dans le but de réduire lincidence de l'hypoxie et des complications cardiaques. ${ }^{3} \mathrm{~A}$ fin des années 1970 , la diffusion des techniques morphiniques à hautes doses $^{4}$ et surtout après l'introduction du fentanyl, ${ }^{5}$ l'assistance respiratoire postopératoire est devenue de rigueur. Récemment, les progrès de la chirurgie, de l'anesthésie, du monitorage et des techniques de circulation extracorporelle ont permis une diminution importante des complications postopératoires. ${ }^{6}$ Ceci nous incite à revoir les indications de l'extubation tardive.

La faisibilité et la sécurité de l'extubation précoce dépend de la méthode anesthésique utilisée pendant l'intervention et de l'analgésie pratiquée après celle-ci. Dernièrement, on a accordé beaucoup d'attention à la technique préconisée par Cheng et al. à Toronto ${ }^{7}$ qui fait appel à des agents de courte durée d'action pour l'in- 
tervention et à l'analgésie à la demande en postopératoire. Ce mois-ci, dans le Journal, Kowalenski et al. ${ }^{8}$ décrivent une technique d'anesthésie générale associée à une rachianesthésie à la bupivacaïne et à la morphine chez dixhuit patients opérés pour revascularisation myocardique. L'objectif de cette technique originale était d'obtenir la stabilité hémodynamique pendant l'intervention, de diminuer le besoin en morphiniques à longue durée d'action et, accessoirement, de bénéficier d'une sympathectomie cardiaque. Après une anesthésie générale à l'alfentanil 97 $\pm 22 \mu \mathrm{g} \cdot \mathrm{kg}^{-1}$ et au midazolam $0,04 \pm 0,02 \mathrm{mg} \cdot \mathrm{kg}^{-1}$, une rachianesthésie (RA) avec de la bupivacaïne hyperbare $23-30 \mathrm{mg}$ ( 15 patients), de la lidocaïne $150 \mathrm{mg}$ (1 patient) ou une combinaison de ces deux agents (2 patients), associée à de la morphine, sans substance préservative, $0,5-1,0 \mathrm{mg}$ a été administrée avec une aiguille $25 \mathrm{G}$ ou $22 \mathrm{G}$. Bien que des patients aient reçu de la phényléphrine à un moment ou un autre de l'intervention, cette administration n'avait pas de relation spécifique avec la RA: en effet, sept patients ont reçu ce vasopresseur après l'induction, huit après la RA, 13 patients pendant la CEC et sept après la CEC). Leurs patients se sont réveillés $1.6 \pm 1,1 \mathrm{~h}$ après leur arrivée aux soins intensifs, Neuf patients $(50 \%)$ ont été extubés le jour même de la chirurgie (en moyenne $6,3 \pm 1,2 \mathrm{~h}$ après celle-ci). Vraisemblablement $75 \%$ de tous les patients auraient pu être extubés le jour même, si on considère que 6 patients sur 18 ont été ventilés pour la nuit en vertu de la pratique systématique qui consistait à ventiler tous les opérés de l'après-midi pour la durée de la nuit. Il est important de noter que cinq patients n'ont pas reçu d'analgésie supplémentaire avant l'extubation. Trois patients ont reçu des perfusions de morphinique pour faciliter la ventilation nocturne et les dix autres ont reçu au besoin des doses totales de morphine de 2 à $14 \mathrm{mg} i v$ ou de fentanyl 100 à $500 \mu \mathrm{g} i \mathrm{v}$.

Les auteurs ont-ils atteint leur objectif? Comme ils le mentionnent, le caractère rétrospectif de leur étude constitue une faiblesse évidente. En effet, il devenait alors impossible de quantifier les changements hémodynamiques et de démontrer qu'on était parvenu à un état de stabilité hémodynamique. Cependant, ils affirment que dans leur institution la coutume est d'administrer de fortes doses de phényléphrine. Comme nous ne possédons pas de groupe contrôle pour établir une comparaison, il nous est difficile de porter un jugement. Ils attestent que l'induction de la RA provoque peu de modifications hémodynamiques supplémentaires mais leur données n'en font pas la preuve. Huit patients sur 18 ont eu besoin de vasopresseurs après la RA. Il est certain que la dose d'alfentanil peropératoire a été moindre que celle que d'autres utilisent. ${ }^{9}$ L'incidence de $16 \%$ de réaction à la sternotomie se compare favorablement à celle de $20 \%$ rapportée par Robbins et al. ${ }^{9}$ surtout si on tient compte que ces derniers ont administré dix fois la dose d'alfentanil. Ceci, ajouté au fait que la concentration d'isoflurane a été gardée très basse $(<0,05 \mathrm{MAC})$, nous permet de croire que la contribution de la rachianesthésie à l'anesthésie a été considérable. On ignore si une sympathectomie cardiaque a été réalisée. Certes, il y a eu un certain degré de bloc sympathique, mais comme le niveau du bloc n'a pas été mesuré nous ne pouvons que spéculer. Malgré ces commentaires négatifs, leurs résultats montrent que cette technique mérite une investigation plus poussée. C'est le caractère rétrospectif de l'étude qui doit être condamné non les résultats. Il faut donc encourager la réalisation d'une étude prospective bien conçue sur le sujet.

Les études antérieures qui font état du bloc nerveux central en chirurgie cardiaque sintéressent à la voie épidurale. ${ }^{10,11}$ On est loin de s'entendre sur l'analgésie épidurale thoracique (AET) pour des patients qui seront complètement héparinés. Les dangers de l'hémorragie et de l'hématome épiduraux avec leur séquelles nerveuses sont surtout redoutés. Jusqu'à maintenant, on n'a pas encore obtenu la réponse définitive sur la question de la sécurité de l'épidurale et de la rachidienne réalisées immédiatement avant l'anticoagulation complète. Cependant, avec l'affluence des publications affirmant la faible incidence des séquelles neurologiques consécutives à ces techniques, elles gagneront en popularité. Une étude de Liem et al. " a comparé l'AET haute pour le traitement postopératoire de la douleur du pontage aorto-coronarien avec l'administration $i v$ intermittente de morphiniques. Pour la prévention des complications neurologiques, les auteurs ont exigé des paramètres coagulatoires normaux. Dans ce but, l'administration d'aspirine a été cessée dix jours avant la chirurgie. De plus, ils ont inséré le cathéter épidural au moins $20 \mathrm{~h}$ avant l'intervention. Les patients du groupe AET se sont réveillés plus rapidement, ont repris une respiration spontanée plus précoce et ont été extubés plus tôt. Leurs résultats sur l'échelle visuelle analogique de la douleur et de sédation ont aussi été supérieurs.

Malgré ses avantages manifestes, cette technique présente deux désavantages majeurs. Premièrement, avec la tendance actuelle qui consiste à diminuer la durée du séjour préopératoire même chez les futurs opérés du coeur, la nécessité d'insérer un cathéter épidural $24 \mathrm{~h}$ à l'avance peut s'avérer impossible dans plusieurs centres hospitaliers. Deuxièmement, plusieurs cardiologues ne seront pas d'accord pour cesser l'aspirine si tôt avant l'intervention, ce qui rend la technique inaccessible à plusieurs patients qui ont besoin d'une chirurgie rapide ou urgente. Un protocole rigoureux et avisé limiterait donc l'usage de l'anesthésie épidurale en chirurgie cardiaque. 
On pourrait spéculer aussi que l'utilisation de l'aiguille rachidienne $27 \mathrm{G}$ plus fine réduirait le risque de formation d'hématome à la période postopératoire comparativement à l'aiguille épidurale 16G. Malheureusement, cet aspect n'a jamais fait l'objet d'études sérieuses. Bien que des études récentes rapportent la sécurité apparente de l'analgésie nerveuse centrale chez des patients anticoagulés, ${ }^{12}$ on ne doit jamais oublier l'éventualité d'un hématome épidural et l'importance de l'examen neurologique répété.

A la recherche perpétuelle de l'anesthésie cardiaque qui assurera la stabilité hémodynamique pendant la période qui précède la $\mathrm{CEC}$, nous sommes peut-être à l'aube d'une nouvelle ère: celle de l'association de l'anesthésie régionale à l'anesthésie générale. L'époque de l'anesthésie cardiaque traditionelle avec les hautes doses de morphiniques semble révolue parce que les analyses de résultats montrent peu de différences entre les techniques anesthésiques: ce qui importe, c'est la maintien de la stabilité hémodynamique peropératoire. Il est à espérer que létude publiée dans ce numéro ${ }^{8}$ stimulera dans l'avenir de la recherche axée sur la sécurité et l'efficacité de cette méthode originale pour la chirurgie des malades cardiaques.

\section{References}

1 Sydown FW. The influence of anesthesia and postoperative analgesic management on lung function. Acta Chirurgica Scandinavica 1988; 550(suppl): 159-68.

2 Prakash $O$, Jonson B, Meji S, et al. Criteria for early extubation after intracardiac surgery in adults. Anesth Analg 1977; 56: 703-8.

3 Lefemine AA, Harken $D E$. Postoperataive care following open heart operations: Routine use of controlled ventialtion. J Thorac Cardiovasc Surg 1966; 52: 207-16.

4 Lowenstein E, Hallowell P, Levine FH, Daggett WM, Austen $W G$, Laver $M B$. Cardiovascular response to large doses of intravenous morphine in man. $\mathrm{N}$ Engl $\mathbf{J}$ Med 1969; 281: 1389-93.

5 Stanley $T H$, Webster $L R$. Anesthetic requirements and cardiovascular effects of fentanyl-oxygen and fentanyldiazepam-oxygen anesthesia in man. Anesth Analg 1978; 57: 411-6.

6 Lell WA, Samuelson PN, Reves JG, Strong SD, et al. Duration of intubation and ICU stay after open heart surgery. South Med J 1979; 72: 773-8.

7 Cheng DCH, Karski J, Peniston C, et al. A prospective randomized controlled clinical trial of early versus conventional tracheal extubation following coronary artery bypass graft (CABG) surgery. Postoperative complications and hospital discharge. Can J Anaesth 1994; 41: A49-A.

8 Kowalewski RJ, MacAdams CL, Eagle CJ, Archer DP, $B$ Baradwaj $B$. Anaesthesia for coronary bypass surgery supplemented with subarachnoid bupivacaine and morphine: A report of 18 cases. Can J Anaesth 1994; 41: 1189-95

9 Robbins GR, Wynands JE, Whalley DG, et al. Pharmacokinetics of alfentanil and clinical responses during cardiac surgery. Can J Anaesth 1990; 37: 52-7.

10 Joachimsson $P-O$, Nystrom $S-O$, Tydén $H$. Early extubation after coronary artery surgery in efficiently rewarmed patients: a postoperative comparison of opioid anesthesia versus inhalational anesthesia and thoracic epidural analgesia. J Cardiothorac Anesth 1989; 3: 444-54.

11 Liem TH, Hasenbos MA, Booij LH, Gielen MJ. Coronary artery bypass grafting using two different anesthetic techniques: part 2: postoperative outcome. J Cardiothorac Vasc Anesth 1992; 6: 156-61.

12 Rao TLK, El-Etr AA. Anticoagulation following placement of epidural and subarachnoid catheters: an evaluation of neurologic sequelae. Anesthesiology 1981; 55: 618-20. 